\title{
Relación entre el crecimiento individual y la abundancia de la población de la sardina del Pacífico Sardinops Caeruleus (Pisces: Clupeidae) (Girard 1856) en Isla de Cedros, Baja California, México
}

\author{
Individual Growth And Population Abundance Relationship In The Pacific Sardine Sardinops \\ Caeruleus (Pisces: Clupeidae) (Girard 1856) From Isla De Cedros, Baja California, Mexico \\ Casimiro Quiñonez-Velázquez, Reyna Alvarado-Castillo y Roberto Félix-Uraga
}

Centro Interdisciplinario de Ciencias Marinas I.P.N., Playa El Conchalito s/n Apdo. Postal 592.

La Paz, Baja California Sur, C.P. 23000, México.

cquinone@ipn.mx

\begin{abstract}
Resumen.- Se analizó el efecto de la abundancia de la población en el crecimiento individual durante la etapa prerecluta de la sardina del Pacífico en Isla de Cedros, Baja California, México. El tamaño de la población se estimó usando un análisis de población virtual y la talla a la edad de los peces se retrocalculó utilizando la relación radio del otolito-longitud estándar. Los resultados mostraron para el primer año de vida de la sardina una mortalidad selectiva por longitud y un crecimiento dependiente de la abundancia de la población. Los peces parcialmente reclutados $(<2$ años de edad) a la pesquería presentaron mayores longitudes retrocalculadas durante su primer año de vida que los peces completamente reclutados ( $>2$ años de edad). La generación de 1980 creció más durante el primer año que las generaciones posteriores (1981-1983); y las diferencias estuvieron asociadas significativamente a la abundancia de la población.

Palabras clave: Sardina del Pacífico, otolitos, edad, crecimiento, retrocálculo de longitud.
\end{abstract}

\begin{abstract}
The effect of the population abundance on the individual growth during the pre-recruit stage of the Pacific sardine from Island de Cedros, Baja California, Mexico was analyzed. The population size was estimated using virtual population analysis and the fish length at age was backcalculate using otolith radius-standard length relationship. During the Pacific sardine first year of life, the results showed a selective mortality by length and a dependent growth of the population abundance. The partially recruited fish $(<2$ years old) to the fishery showed larger backcalculate length during their first year old than fully recruited fish ( $>2$ years old). The 1980 year class grew more during your first year of life than later year classes (1981-1983); and the differences were significantly associated with the population abundance.

Key words: Pacific sardine, age, otolith, backcalculate length.
\end{abstract}

\section{Introducción}

La sardina del Pacífico Sardinops caeruleus (Girad 1856) se distribuye en el Océano Pacífico nororiental, desde el sureste de Alaska hasta el sur del Golfo de California (Parrish et al. 1989). Esta especie fue explotada comercialmente en las costas de California, EUA, obteniéndose la captura máxima de 800 mil ton, en 1936 y a partir de ese año se observó una rápida disminución de los rendimientos hasta que en 1967 la pesquería se cerró (Radovich 1982). Murphy (1966), señaló que la presión de la pesca redujo el potencial reproductivo de la población a niveles en los que el colapso fue inevitable. Por otro lado, Iles (1973), analizando el crecimiento del grupo de edad cero de la sardina del Pacífico del sur de California durante el periodo 1934 a 1955, detectó que los cambios en longitud durante el primer año de vida se asociaron a cambios en el reclutamiento, resultando una alternativa mas expedita que analizar datos de captura y esfuerzo (Murphy 1966, Hampton \& Majkowski 1986).

La pesca de sardina del Pacífico se ha desarrollado en la costa occidental de Baja California desde 1940, como una consecuencia de la disminución de los rendimientos en la del sur de California. En sus inicios la pesquería se desarrollaba entre Ensenada e Isla de Cedros. A fines de los 60 la pesca ya incluía al Golfo de California, donde a la fecha se obtienen los mayores rendimientos (Cisneros-Mata et al. 1995). En la periferia de Isla de Cedros, la abundancia relativa de la sardina 
se incrementó de aproximadamente $27 \mathrm{t}$ de CPUE en 1960, a un máximo de 65.5 t de CPUE en 1967. El tamaño relativo del stock se mantuvo desde entonces en 25 t de CPUE, hasta que a fines de 1994 la planta procesadora en la isla cerró por problemas operativos.

En algunas especies de peces pelágicos se ha identificado que el crecimiento individual durante el primer año de vida es afectado por la abundancia de la población (Iles 1968, 1973; Overholtz 1989). En la costa occidental de Baja California, la sardina del Pacífico presenta tasas anuales de crecimiento $(\mathrm{k})$ de 0.99 a 1.19 (Félix-Uraga \& Félix-Uraga 1987, FélixUraga 1990, Alvarado-Castillo \& Félix-Uraga 1996, Butler et al. 1996). Para esta zona, no se han desarrollado estudios sobre el crecimiento de la sardina del Pacífico durante su primer año de vida (etapa prerecluta). Al respecto, si la longitud, durante esa etapa del desarrollo, fuera dependiente de la abundancia poblacional (Iles 1973), un análisis de su variabilidad podría ser de utilidad en la estimación relativa de la población, una de las principales características para establecer estrategias de explotación del recurso (Cushing \& Harris 1973, Overholtz et al. 1991).

La sardina del Pacífico se recluta completamente a la pesquería a los 2 años (173£16 mm LE) (Félix-Uraga 1992) y la pesca capturará una muestra sesgada de peces de edad uno, siendo los peces más grandes de esta edad susceptibles a la pesca. Por lo tanto un estudio de la variabilidad de la longitud durante el primer año de vida deberá considerar la estimación de la longitud a esa edad a partir de una función que relacione la longitud del pez con alguna estructura del cuerpo. Además, si existe algún efecto de mortalidad por pesca relacionado con la longitud del pez (Lee 1912), el retrocálculo de la longitud al primer año dependerá de la edad del pez sobre el cual se hicieron las estimaciones (Iles 1968). Por tanto, un análisis del crecimiento a la edad deberá considerar este efecto potencial de la mortalidad en función de la longitud del pez.

No obstante que se ha reconocido la importancia del crecimiento individual en la dinámica de la población (Iles 1968, De Anda-Montañez et al. 1999), pocos estudios han evaluado la consecuencia de la variación en la tasa de crecimiento de los peces durante la etapa juvenil (Francis 1993, Sogard 1997). Las evidencias sugieren que un rápido crecimiento puede ser ventajoso para la supervivencia durante las primeras etapas del desarrollo en los peces (Houde 1987, Anderson 1988, Fortier y Quiñónez-Velázquez 1998), pero es importante distinguir entre los cambios en la distribución de tallas asociados a la mortalidad de los cambios debido al crecimiento individual. Este problema resultará del reclutamiento parcial al stock explotado de los peces con un crecimiento más rápido, que los peces de la misma edad con crecimiento menor y tendrá un efecto de sobre estimación de la talla promedio de los peces de la cohorte parcialmente reclutada a la población.

En este trabajo examinamos, el efecto de la abundancia de la población sobre el crecimiento individual de los prerreclutas, la mortalidad por pesca asociada a la talla y se describe el patrón general de crecimiento de la sardina del Pacífico en Isla de Cedros, Baja California, México.

\section{Materiales y Métodos}

No obstante que la pesca comercial de sardina del Pacífico, en la periferia de Isla de Cedros, B.C., se realizó desde la década de los años 40 , se tienen datos de captura y esfuerzo desde 1960 los cuales se obtuvieron de los registros de la planta procesadora en la Isla. La información biológica se obtuvo de muestreos mensuales de la descarga comercial de sardina en el muelle de la Isla, durante 1985 y 1986 (Fig. 1). El muestreo consistió en medir la longitud estándar (LE) de 100 o más individuos elegidos al azar, anotando la frecuencia de aparición en intervalos de $5 \mathrm{~mm}$. Para la determinación de la edad, de cada intervalo de longitud se escogieron los primeros cinco individuos (Félix-Uraga 1986), este tipo de submuestreo garantiza incluir representantes de toda la estructura de longitudes.

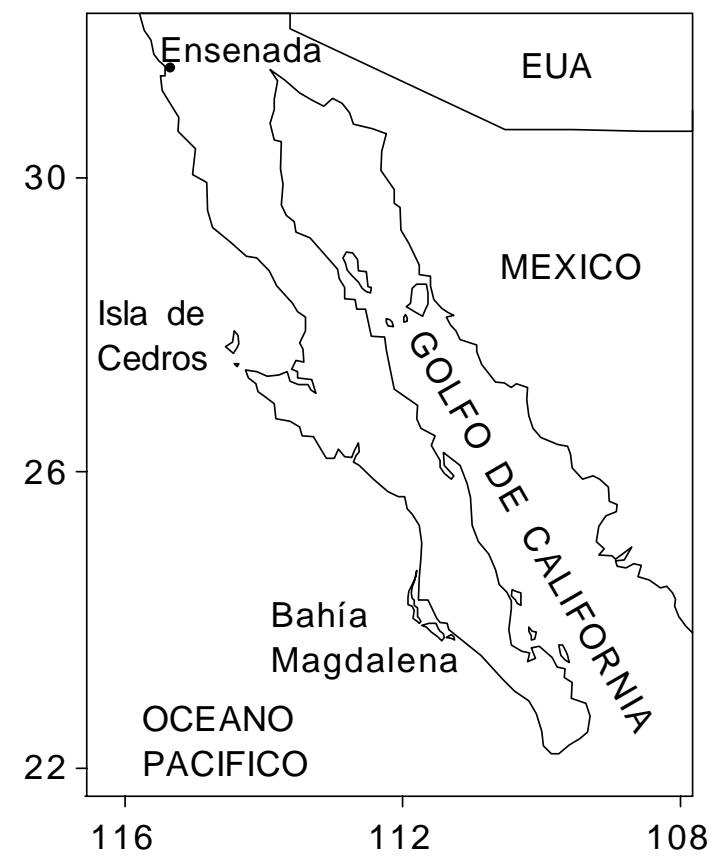

Figura 1

Costa occidental de Baja California. Se presenta la zona de estudio y las principales zonas de pesca de sardina del Pacífico.

Western coast of Baja California. Study area and Pacific sardine main fishing areas. 
En el laboratorio los peces de la submuestra fueron pesados (peso total $\pm 1 \mathrm{~g}$ ), se les medió la LE $( \pm 1 \mathrm{~mm}$ ) y se les extrajeron los otolitos (sagittae), para posteriormente determinar la edad. Los otolitos se lavaron con agua y jabón, se montaron sobre portaobjetos con resina sintética (Protexx) y se leyeron con la ayuda de un microscopio estereoscópico y luz reflejada, sobre un fondo oscuro. Bajo estas condiciones se observaron zonas blancas (opacas) y obscuras (hialinas). La edad se determinó contando el número de bandas de crecimiento en los otolitos, representadas por una zona opaca y una traslucida (Fitch 1951). Para validar la naturaleza temporal de la formación de las bandas de crecimiento, se graficó por estación del año el porcentaje de otolitos con margen opaco y el ancho de la banda marginal. El ancho de la banda marginal consistió en la distancia desde la ultima banda completamente depositada al borde del otolito. Para demostrar la proporcionalidad del crecimiento somático y del otolito se ajustó una regresión funcional (Ricker 1973) a los datos radio del otolito-LE.

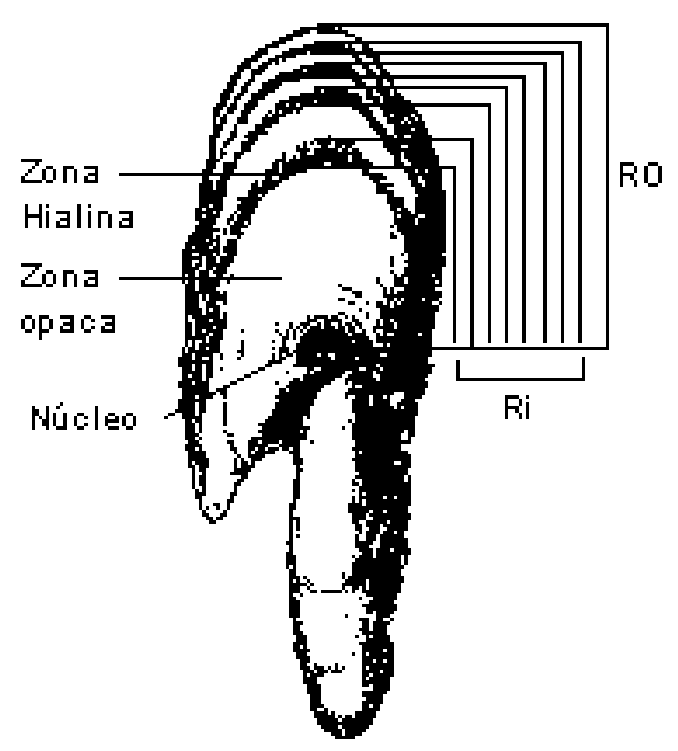

Figura 2

Representación esquemática de un otolito de sardina del Pacífico y las diferentes medidas registradas.

Pacific sardine otolith schematic representation and different measurements.

Para explorar la relación entre el crecimiento durante el primer año de vida y la abundancia relativa de la población de la sardina del Pacífico en Isla de Cedros, se seleccionaron al azar, de la submuestra mensual, hasta 5 peces por grupo de edad y como índice de abundancia de la sardina se utilizó el número de peces estimados a través un análisis de población virtual realizado con el programa FISAT (Gayanilo et al. 1995) utilizando la matriz estructurada por edades de la captura de sardina en Bahía Magdalena (Félix-Uraga et al. 1996). A los otolitos seleccionados $(\mathrm{n}=170)$ se les midió el radio $(\mathrm{RO} \pm$ $1 \mu \mathrm{m}$ ), desde el núcleo al margen posterior, y el radio a cada zona de crecimiento $\left(\mathrm{R}_{\mathrm{i}} \pm 1 \mu \mathrm{m}\right)$ (Fig. 2). Las mediciones se efectuaron con la ayuda de un sistema video-digitalizador, utilizando el programa de computadora SigmaScan Pro (Jandel Scientific, San Rafael, CA 94912-7005). Utilizando la relación RO-LE se retrocalcularon las longitudes del pez a cada banda de crecimiento y a estos datos (edad-LE) se les ajusto el modelo de crecimiento de von Bertalanffly usando el modulo NONLINEAR del programa de computadora STATISTICA (StatSoft 1995).

\section{Resultados}

\section{Edad}

Para la determinación de la edad se colectaron otolitos de 865 peces (474 en 1985 y 391 en 1986). Se identificaron cinco grupos de edad, $0+$ a $4+$ grupos con tallas entre 147 a $236 \mathrm{~mm}$ de LE. El porcentaje estacional de otolitos con margen opaco indica que se forma una banda de crecimiento en el transcurso de un año (Fig. 3a). El porcentaje fue bajo durante otoñoinvierno y máximo en primavera-verano, sugiriendo que durante el invierno se deposita la zona traslucida y se completa una banda de crecimiento. Lo anterior se fortalece al apreciar el cambio estacional del ancho de la banda de crecimiento marginal en los otolitos del grupo2 de edad (Fig. 3b), el valor máximo fue en invierno confirmando la periodicidad anual en la formación de las bandas de crecimiento. La asignación de edad fue en años y correspondió al número de bandas completamente depositadas en los otolitos. La justificación para el uso de los otolitos en la determinación de edad y crecimiento de la sardina del Pacífico es el constante incremento del promedio de la distancia a cada zona opaca para peces de todas las edades (Tabla 1) y la significativa relación entre el RO y la LE (Fig. 4):

$$
\text { LE=1.58+RO*123.98 }(R=0.69, n=170, P<0.001)
$$

Esta ecuación describe la relación entre RO y LE, asumiendo que no existe desviación de las mediciones individuales respecto a la línea de regresión. Para fines de retrocálculo, la regresión se modificó de acuerdo al procedimiento de Fraser-Lee (Carlander 1981), para incorporar las diferencias de cada pez respecto a la línea de regresión, resultando la siguiente ecuación: 

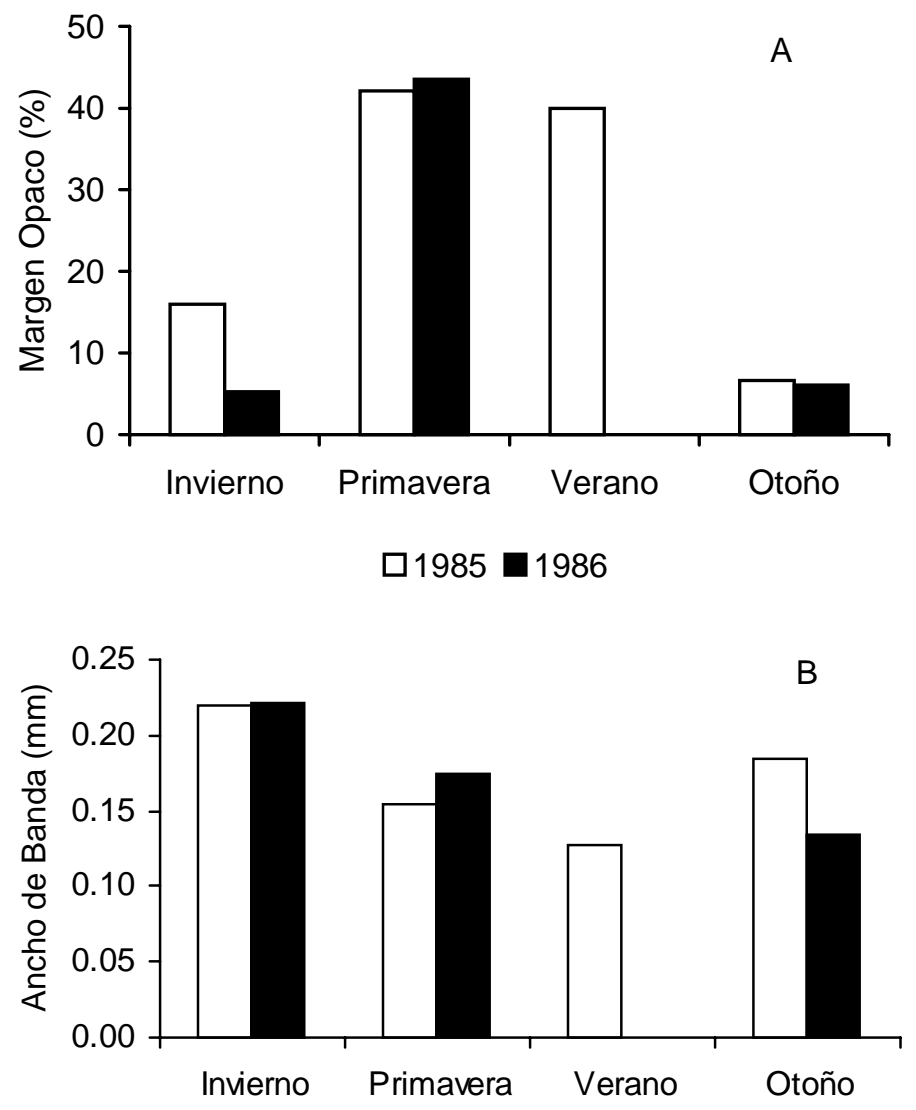

Figura 3

A) Porcentaje estacional de otolitos con margen opaco, y B) ancho de la banda de crecimiento marginal del otolito del grupo-2 de edad de la sardina del Pacífico.

A. Seasonal percentage of opaque margin otolith, and B. Wide of the 0group marginal growth band otolith of the Pacific sardine.

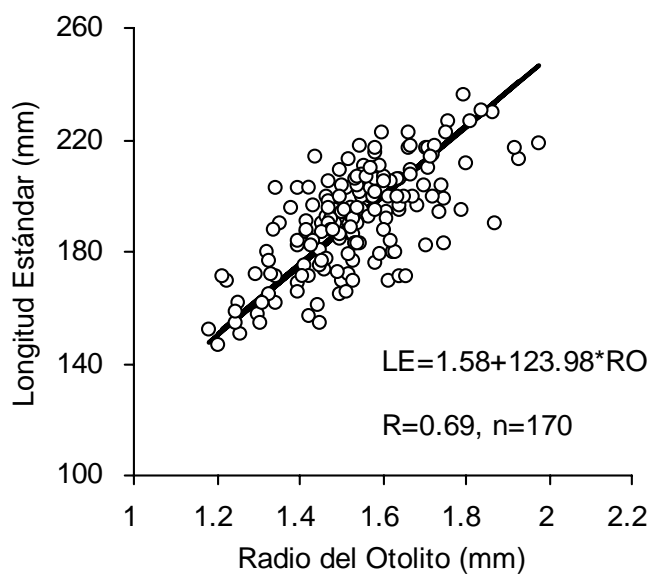

Figura 4

Relación radio del otolito (RO) y longitud estandar (LE) de la sardina del Pacífico.

Otolith radius (RO) and standard length (LE) relationship of Pacific sardine.

$$
\mathrm{LE}_{\mathrm{i}}=1.58+\mathrm{R}_{\mathrm{i}} *\left(\mathrm{LE}_{\mathrm{j}}-1.58\right) / \mathrm{RO}_{\mathrm{j}}
$$

donde $\mathrm{R}_{\mathrm{i}}$ es el radio del otolito a una edad $\mathrm{i}$, $L E_{i}$ es la longitud estimada por retrocálculo a la edad $\mathrm{i}, \mathrm{LE}_{\mathrm{j}} \mathrm{y} \mathrm{RO}_{\mathrm{j}}$ son la longitud del pez $\mathrm{y}$ radio $_{\mathrm{j}}$ del otolito al momento de la captura.

\section{Mortalidad Dependiente de la Longitud}

Comparando la longitud retrocalculada a los seis meses $\mathrm{y}$ al año de vida, $\mathrm{LE}_{0.5}$ primer zona opaca y $\mathrm{LE}_{1}$ primer zona traslucida respectivamente, a partir de peces de diferentes edades, fue posible identificar la presencia de mortalidad relacionada con la longitud. Las longitud $\mathrm{LE}_{0.5} \quad \mathrm{y} \quad \mathrm{LE}_{1}$ retrocalculadas de sardinas de $2+$ fue menor que las longitudes estimadas con individuos de $0+$ y 1+ (Tabla 2). La comparación múltiple del promedio de la longitud $\mathrm{LE}_{0.5} \mathrm{y}$ $\mathrm{LE}_{1}$ por edad indica, de manera general, que no hubo diferencia significativa entre los individuos de edad de $2+$ a $4+$ años ( $p>0.05$ ). Sin embargo, la diferencia fue significativa entre los individuos de edad $0+$ y $1+$ años $(\mathrm{p}<0.05)$. Esto indica que para fines de describir el crecimiento individual durante el primer año de vida de la sardina del Pacífico se deben considerar los peces completamente reclutados a la pesquería. Esto es debido a que las tallas de los peces < 2 años de edad en la captura, estarán poco representadas y sesgadas hacia las tallas mayores de su generación.

\section{Efecto de la Abundancia de la Población en el Crecimiento}

Para el retrocálculo de la longitud a la edad, los peces $>2$ años de edad se agruparon para incrementar el tamaño de la muestra y obtener estimaciones mas precisas (Tabla 3). Comparando el promedio de la longitud retrocalculada a la edad, por generación, se observa que la sardina del Pacífico de la generación 1980 mostró un mayor crecimiento durante los primeros seis meses $\left(\mathrm{LE}_{0.5}\right)$ que las generaciones posteriores. Sin embargo, previo a la edad de reclutamiento $\left(\mathrm{LE}_{1.5}\right)$ las diferencias en longitud se invirtieron y se detectó una fuerte relación entre el promedio de $L_{1.5}$ y el promedio de la longitud a la edad de reclutamiento $\mathrm{LE}_{2}$ $\left(r^{2}=0.98\right)$. 


\section{Tabla 1}

Intervalo, promedio y desviación estándar del radio del otolito (mm) a la zona opaca de cada banda de crecimiento de la sardina del Pacífico. Promedio en negritas, desviación estándar en paréntesis y número de peces (n).

Range, average and standard deviation $(\mathrm{mm})$ of opaque zone otolith radius from each growth band of Pacific sardine. Average in bold, standard deviation in parenthesis and fish number (n).

\begin{tabular}{|c|c|c|c|c|c|c|}
\hline \multirow{2}{*}{$\begin{array}{l}\text { Edad } \\
\text { a la } \\
\text { captura }\end{array}$} & \multirow[b]{2}{*}{$\mathrm{n}$} & \multicolumn{5}{|c|}{$\begin{array}{c}\text { Edad } \\
\text { (años) }\end{array}$} \\
\hline & & 0 & 1 & 2 & 3 & 4 \\
\hline \multirow[t]{2}{*}{0} & 14 & $1.02-1.41$ & & & & \\
\hline & & $1.2(0.11)$ & & & & \\
\hline \multirow[t]{2}{*}{1} & 52 & $0.70-1.51$ & $1.11-1.72$ & & & \\
\hline & & 1.1(0.16) & $\mathbf{1 . 4}(0.12)$ & & & \\
\hline \multirow[t]{2}{*}{2} & 53 & $0.66-1.35$ & $1.03-1.61$ & $1.28-1.77$ & & \\
\hline & & 1.0(0.20) & $1.3(0.13)$ & 1.5(0.11) & & \\
\hline \multirow[t]{2}{*}{3} & 39 & $0.62-1.36$ & $1.02-1.58$ & $1.19-1.68$ & $1.28-1.85$ & \\
\hline & & $\mathbf{1 . 0}(0.17)$ & $1.2(0.14)$ & $\mathbf{1 . 4}(0.12)$ & 1.5(0.12) & \\
\hline \multirow[t]{2}{*}{4} & 12 & $0.70-1.41$ & $0.97-1.59$ & $1.11-1.72$ & $1.25-1.78$ & $1.42-1.90$ \\
\hline & & 1.1(0.23) & 1.3(0.18) & 1.5(0.17) & $1.6(0.15)$ & 1.7(0.13) \\
\hline
\end{tabular}

Tabla 2

Resultados de la comparación múltiple (ANOVA) de la longitud $\mathrm{LE}_{0.5}$ y $\mathrm{LE}_{1}$ retrocalculada a partir de diferentes edades. Las longitudes se estimaron a través la relación RO-LE.

Multiple comparison (ANOVA) results of backcalculate $\mathrm{LE}_{0.5}$ and $\mathrm{LE}_{1}$ from different ages. Lengths were estimated from RO-LE relationship.

\begin{tabular}{|c|c|c|c|}
\hline \multirow[b]{2}{*}{ Edad } & \multicolumn{3}{|l|}{$\mathrm{LE}_{0.5}$} \\
\hline & $\mathrm{n}$ & Valor F & Probabilidad \\
\hline 0 vs 1 & 65 & 9.50 & $* *$ \\
\hline 0 vs 2 & 66 & 19.24 & ** \\
\hline 0 vs 3 & 52 & 20.14 & $* *$ \\
\hline 0 vs 4 & 25 & 9.39 & $* *$ \\
\hline 1 vs 2 & 104 & 5.98 & $*$ \\
\hline 1 vs 3 & 90 & 5.04 & $*$ \\
\hline 1 vs 4 & 63 & 0.20 & n.s \\
\hline 2 vs 3 & 91 & 0.01 & n.s \\
\hline 2 vs 4 & 64 & 1.07 & n.s \\
\hline \multirow[t]{2}{*}{3 vs 4} & 50 & 1.00 & n.s \\
\hline & $\mathrm{LE}_{1}$ & & \\
\hline 1 vs 2 & 104 & 10.42 & $* *$ \\
\hline 1 vs 3 & 90 & 10.08 & $* *$ \\
\hline 1 vs 4 & 63 & 1.61 & n.s \\
\hline 2 vs 3 & 91 & 0.01 & n.s \\
\hline 2 vs 4 & 64 & 0.56 & n.s \\
\hline 3 vs 4 & 50 & 0.67 & n.s \\
\hline
\end{tabular}

Tabla 3

Promedio de la longitud $(\mathrm{mm})$ durante la etapa pre-recluta (<2 años) de la sardina del Pacífico en Isla de Cedros. Las longitudes se estimaron a través la relación RO-LE. Desviación estándar en paréntesis.

Average length (mm) during pre-recruit stage $(<2$ years) of Pacific sardine in Isla de Cedros. Lengths were estimated from RO-LE relationship. Standard deviation in parenthesis.

\begin{tabular}{ccccc}
\hline $\begin{array}{c}\text { Clase } \\
\text { Anual }\end{array}$ & $\mathrm{LE}_{0.5}$ & $\mathrm{LE}_{1}$ & $\mathrm{LE}_{1.5}$ & $\mathrm{LE}_{2}$ \\
\hline 1980 & $129.51(14.53)$ & $137.23(14.48)$ & $147.34(15.31)$ & $157.61(17.95)$ \\
1981 & $119.79(23.12)$ & $130.64(21.10)$ & $151.08(13.75)$ & $160.09(12.55)$ \\
1982 & $124.91(25.22)$ & $139.25(25.08)$ & $157.94(18.30)$ & $167.74(17.41)$ \\
1983 & $128.89(22.01)$ & $141.84(22.01)$ & $168.07(14.46)$ & $180.23(11.99)$
\end{tabular}
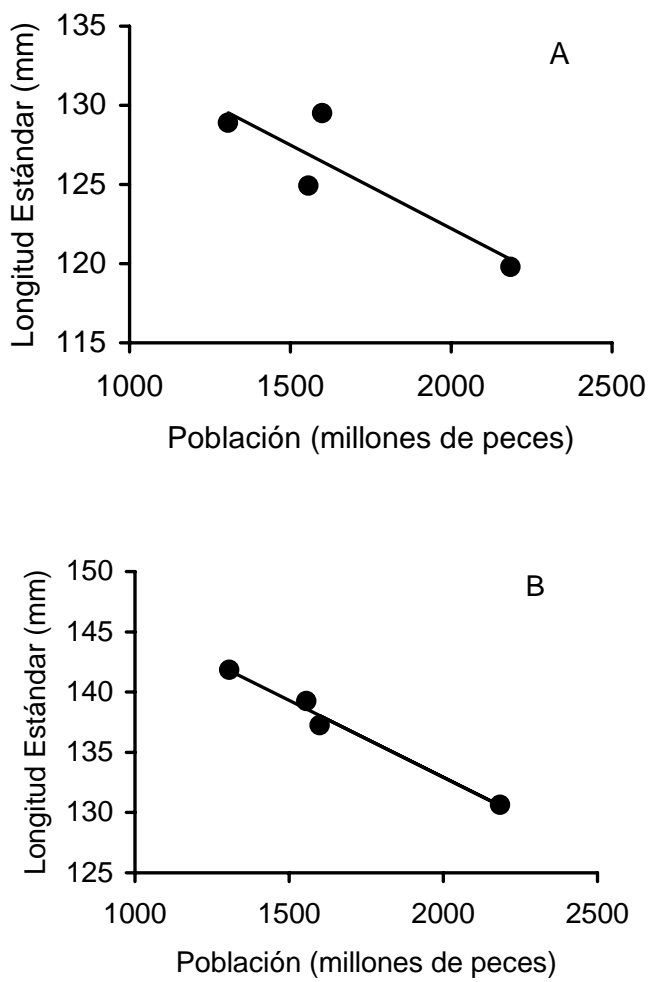

Figura 5

Relación entre la abundancia relativa de la población de sardina del Pacífico y el crecimiento individual durante el primer año de vida. A) Longitud estándar retrocalculada a los seis meses de edad $L_{0.5}$, B) Longitud estándar retrocalculada al año de edad $L E_{1}$.

Relationship of population relative abundance and first year individual growth of Pacific sardine. A) Backcalculated standard length at six months old $\mathrm{LE}_{0.5}$, and B) Backcalculated standard length at one year old $\mathrm{LE}_{1}$. 
Para evaluar el efecto de la abundancia de la población en el crecimiento de la sardina del Pacífico durante su primer año de vida, se aplicó una regresión lineal a los datos del tamaño de la población de sardina en número de peces (1980-1983) contra las longitudes $\mathrm{LE}_{0.5}$ y $\mathrm{LE}_{1}$ por generación (Fig. 5). La evidencia estadística sugiere que la longitud a los seis meses de vida $\left(\mathrm{LE}_{0.5}\right)$ de una generación es independiente de la abundancia de la población (Fig. 5a) $\left(\mathrm{r}^{2}=0.77, \mathrm{P}=0.12\right)$, pero la longitud al año de edad $\left(\mathrm{LE}_{1}\right)$ estuvo fuertemente influenciada por la abundancia (Fig. 5b) $\left(r^{2}=0.98\right.$, $\mathrm{P}=0.01$ ). Valores mayores de $\mathrm{LE}_{1}$ se asocian a valores relativamente bajos de la población.

Para describir el patrón general del crecimiento individual, a los datos de longitud retrocalculada a la edad se ajusto el modelo de von Bertalanffly. Los valores de los parámetros fueron $\mathrm{L} \infty=221.03 \mathrm{~mm}, \mathrm{k}=$ 0.41/año y t $\mathrm{y}_{0}=-1.46 /$ años. De acuerdo a estos valores, los peces a los dos años de edad alcanzarán el 75\% de la longitud infinita.

\section{Discusión}

La sardina del Pacífico de Isla de Cedros, deposita cada año una zona opaca y una zona traslucida. Este patrón estacional ha sido validado para la sardina del Pacífico en el sur de California (Barnes \& Foreman 1994), en la macarela (Scomber japonicus) (Gluyas-MIllán \& FelixUraga 1990) y en el charrito (Trachurus symmetricus) (Knaggs \& Sunada 1973). A través del cambio estacional del ancho de la banda de crecimiento marginal en los otolitos se evidenció que las zonas opacas reflejan periodos de crecimiento rápido, correspondiendo a los meses de primavera verano cuando el alimento es abundante, y las zonas traslúcidas a periodos de lento crecimiento (Beckman \& Wilson 1995).

A causa de un reclutamiento parcial, los retrocálculos de $\mathrm{LE}_{0.5}$ y $\mathrm{LE}_{1}$ de sardinas $<2$ años de edad mostraron un sesgo positivo respecto a los retrocálculos con sardinas de >2 años de edad. El análisis de la longitud estimada por retrocálculo a la edad a partir de muestras de la captura comercial de la sardina del Pacífico en Isla de Cedros, presenta evidencia de una mortalidad por pesca selectiva por longitud en los primeros grupos de edad $(0+$ y $1+)$. Esto significa que para la estimación del crecimiento durante el primer año de vida, se deben considerar las edades completamente reclutadas a la pesquería. Si únicamente los peces mas grandes de un grupo de edad son muestreados, el crecimiento para esas edades será sobrestimado. Lo anterior también fue detectado por Iles (1968) para el arenque (Clupea harengus) y por Beacham (1981) para el bacalao (Gadus morhua) en el sur del Golfo de St.
Lawrence. En conclusión, cuando una población de peces es explotada, los peces de una generación, con rápido crecimiento serán vulnerables a la pesca antes que los peces con lento crecimiento, resultado de la selectividad del arte de pesca y/o comportamiento de la especie (MacCall 1985, Fréon \& Misund 1999).

Las diferencias en el crecimiento de la sardina del Pacífico durante su primer año de vida (Tabla 3) sugieren que el tamaño de la población afecta el crecimiento. Los resultados muestran que la $\mathrm{LE}_{1}$ esta fuertemente influenciada por la población. Overholtz (1989) presenta evidencias para la macarela del Atlántico (Scomber scombrus), de un crecimiento dependiente de la densidad de la población. Marr (1960), analizando los datos de la sardina del Pacífico en el sur de California, EUA, para el periodo 1934 a 1955, encontró una relación inversa entre la talla al primer año de vida y el tamaño de la generación. Iles (1973), reanalizando los datos para la sardina del Pacífico durante 1934 a 1955, concluye que las mayores tallas promedio al primer año de vida de las generaciones posterior a 1943 fue el resultado de la reducción por pesca de la población de peces adultos y que el colapso de la población fue evidente desde 1945.

Una explicación alternativa del patrón de crecimiento observado en la sardina del Pacífico de Isla de Cedros, podría encontrase en el régimen anual de la temperatura superficial del mar (TSM), régimen que esta relacionado con la presencia del fenómeno El Niño, y en particular durante el periodo de estudio la zona estuvo influenciada por El Niño 1982-83 (Lenarz et al. 1995). Sin embargo, la relación entre la TSM y el patrón de crecimiento no fue significativa $(\mathrm{P}>0.05)$. FélixUraga et al. (1996) y Alvarado-Castillo \& Félix-Uraga (1997) señalan que la sardina del Pacífico en la costa occidental de Baja California presenta desplazamientos estacionales asociados a la TSM. Comentan, que los desplazamientos son más intensos (distancia y duración) durante la presencia de condiciones anómalas asociadas al evento El Niño. Este comportamiento pudo haber permitido a las sardinas evadir las condiciones de menor productividad durante 1983 desplazándose hacia zonas mas al norte.

\section{Agradecimientos}

Este estudio fue apoyado por la CGPI (Coordinación General de Posgrado e Investigación) del Instituto Politécnico Nacional. Los autores agradecen los comentarios de María Georgina Gluyas-Millán, Jon Elorduy-Garay y a dos revisores anónimos cuyos comentarios permitieron mejorar el manuscrito. Los autores son miembros de COFAA, EDI del IPN. 


\section{Literatura Citada}

Alvarado-Castillo RM \& R Félix-Uraga. 1996. Edad y crecimiento de la sardina Monterrey Sardinops caeruleus (PISCES:CLUPEIDAE) en Isla de Cedros, Baja California, México, durante 1985 y 1986. Boletín de Investigaciones Marinas y Costeras 25: 77-86.

Alvarado-Castillo RM \& R Félix-Uraga. 1997. Modelo de la distribución anual de la sardina del Pacífico Sardinops caeruleus en la costa oeste de la península de Baja California. Informe Técnico 1995 del Taller de Pelágicos Menores. CIBNOR-La Paz, Baja California Sur, México.

Anderson JT. 1988. A review of size dependent survival during pre-recruit stages of fishes in relation to recruitment. Journal of Northwest Atlantic Fishery Science 8: 55-66.

Barnes JT and TJ Foreman. 1994. Recent evidence for the formation of annual growth increments in the otoliths of young Pacific sardines (Sardinops sagax). California Fish and Game 80: 29-35.

Beckman DW \& CA Wilson. 1995. Seasonal timing of opaque zone formation in fish otoliths. En: Secor, DH, JM Dean \& SE Campana (eds), Recent developments in fish otolith research 27-43. University of South Carolina Press.

Beacham TD. 1981. Variability in growth during the first 3 years of life of cod (Gadus morhua) in the southern Gulf of St. Lawrence. Canadian Journal of Zoology 59: 614620.

Butler JL, ML Granados, JT Barnes, M Yaremko \& BJ Macewics. 1996. Age composition, growth, and maturation of the Pacific sardine (Sardinops sagax) during 1994. California Cooperative Oceanic Fisheries Investigations Reports 37: 152-159

Carlander KD. 1981. Caution on the use of the regression method of backcalculating lengths from scale measurements. Fisheries 6:2-4.

Cisneros-Mata MA, MO Nevárez-Martínez \& MG Hammann. 1995. The rise and fall of the Pacific sardine, Sardinops sagax caeruleus GIRARD, in the Gulf of California, Mexico. California Cooperative Oceanic Fisheries Investigations Reports 36:136-142.

Cushing DH \& JGK Harris. 1973. Stock and recruitment and the problem of density dependence. Rapports et ProcèsVerbaux des Réunions du Conseil International pour l'exploration de la Mer 164: 142-155.

De Anda-Montañez A, F Arreguin-Sánchez \& S MartínezAguilar. 1999. Length-based growth estimates for Pacific sardine (Sardinops sagax) in the Gulf of California, Mexico. California Cooperative Oceanic Fisheries Investigations Reports 40:179-183.

Félix-Uraga R. 1986. Edad, crecimiento y estructura poblacional de Sardinops sagax caerulea en Bahía Magdalena, durante 1981 a 1984. La Paz, B.C.S., México, Tesis de Maestría Centro Interdisciplinario de Ciencias Marinas-IPN 103 p
Félix-Uraga R. 1990. Crecimiento de Sardinops sagax caerulea en Bahía Magdalena, México. Investigaciones Marinas CICIMAR 5: 27-31.

Félix-Uraga R. 1992. Composición por edades de la sardina Monterrey Sardinops caeruleus capturada en Bahía Magdalena, B.C.S., México, de 1981 a 1991. Investigaciones Marinas CICIMAR 7: 9-13.

Félix-Uraga R \& F Félix-Uraga. 1987. Estructura por tallas y edades de la captura de sardina Monterrey en Bahía Magdalena, durante 1985-1986. 35-37. En: RE RamírezRodríguez (ed), Memorias del Simposium sobre Investigación Biológica, Oceanográfica y Pesquera en México 35-37. La Paz, Baja California Sur, México.

Félix-Uraga R, RM Alvarado-Castillo \& R Carmona-Piña. 1996. The sardine fishery along the western coast of Baja California, 1981 to 1994. California Cooperative Oceanic Fisheries Investigations Reports 37: 188-192.

Fitch JE. 1951. Age composition of the southern California catch of Pacific mackerel 1939-40 through 1950-51. California Department of Fish and Game, Fishery Bulletin 83: 1-73.

Fortier L \& C Quiñonez-Velázquez. 1998. Dependence of survival on growth in larval pollock Pollachius virens and haddock Melanogrammus aeglefinus: a field study based on individual hatchdates. Marine Ecology Progress Series 174: 1-12.

Francis MP. 1993. Does water temperature determine year class strength in New Zealand snapper (Pagrus auratus, Sparidae)?. Fisheries Oceanography 2: 65-72.

Fréon P \& OA Misund. 1999. Dynamics of pelagic fish distribution and behaviour: effects of fisheries and stock assessment, 348 p. Fishing News Books, Oxford.

Gayanilo FC Jr, P Sparre \& D Pauly. 1995. The FAOICLARM Stock Assessment Tools (FISAT) User's Guide. FAO Computerized Information Series (Fisheries). No. 8. Rome, FAO.

Gluyas-Millán MG \& R Felix-Uraga. 1990. Periodicidad de las bandas de crecimiento en otolitos de la macarela Scomber japonicus de Bahía Vizcaíno, México. Investigaciones Marinas CICIMAR 5: 33-36.

Hampton J \& J Majkowski. 1986. Scientists fear SBT problems worsening. Australian Fisheries 45: 6-9.

Houde ED. 1987. Fish early life dynamics and recruitment variability. American Fisheries Society Symposium 2: 1729.

Iles TD. 1968. Growth studies on North Sea herring II. 0group growth of east anglian herring. Journal du Conseil Permanent pour l'Exploration de la Mer 32: 98-116.

Iles TD. 1973. Interaction of environment and parent stock size in determining recruitment in the Pacific sardine as revealed by analysis of density-dependent 0 -group growth. Procès-Verbaux des Réunions du Conseil International pour l'exploration de la Mer 164: 228-240. 
Knaggs EH \& JS Sunada. 1973. Validity of otolith age determinations for jack mackerel, Trachurus symmetricus, from the southern California bight area. California Department of Fish and Game, Marine Resources Technical Reports 21: 1-11.

Lee RM. 1912. An investigation into the methods of growth determination in fishes. Conseil international pour l'Exploration de la Mer, Publication Circonstance No. 63.

Lenarz WH, D Ventresca, WM Graham, FB Schwing \& F Chavez. 1995. Explorations of the El Niño events and associated biological population dynamics off Central California. California Cooperative Oceanic Fisheries Investigations Reports 36: 106-118.

MacCall AD. 1985. Informe del grupo de trabajo sobre investigación y monitoreo de recursos. En: Csirke, J \& GD Sharp (eds), Informes de la consulta de expertos para examinar los cambios en la abundancia y composición por especies de recursos de peces neríticos FAO Informe de Pesca (291) 1: 104 p.

Marr JC. 1960. The causes of major variations in the catch of the Pacific sardine Sardinops caerulea. Proc. World Scientific meeting of the Biology of Sardines and Related Species, 3:667-791, FAO, Rome.

Murphy GY. 1966. Population biology of the Pacific sardine (Sardinops caerulea). Proceedings of the California Academy of Science Series 4, 34: 84 pp.
Overholtz WJ. 1989. Density dependent growth in the Northwest Atlantic stock of Atlantic mackerel. Journal of Northwest Atlantic Fishery Sciences 9: 127-135.

Overholtz WJ, SA Murawski \& WL Michaels. 1991. Impact of compensatory responses on assessment advice for Northwest Atlantic mackerel stock. Fishery Bulletin 89: 117-128.

Parrish RH, R Serra \& WS Grant. 1989. The monotypic sardines, Sardina and Sardinops: Their taxonomy, distribution, stock stucture, and zoogeography. Canadian Journal of Fisheries and Aquatic Sciences 46: 2019-2036.

Radovich J. 1982. The collapse of the California sardine fishery. What have we learned?. California Cooperative Oceanic Fisheries Investigations Reports 23: 56-77.

Ricker WE. 1973. Linear regressions in fisheries research. Journal of the Fisheries Research Board of Canada 30: 409-434.

Sogard SM. 1997. Size-selective mortality in the juvenile stage of teleost fishes: a review. Bulletin of Marine Science 60: 1129-1157.

StatSoft Inc. 1995. STATISTICA for Windows [Computer program manual]. Tulsa, OK. 